EPJ Web of Conferences 59, 05007 (2013)

DOI: $10.1051 /$ epjconf/20135905007

(C) Owned by the authors, published by EDP Sciences, 2013

\title{
Multi-dimensional PIC-simulations of parametric instabilities for shock-ignition conditions
}

\author{
C. Riconda ${ }^{1, a}$, S. Weber ${ }^{1}$, O. Klimo ${ }^{2}$, A. Héron ${ }^{3}$ and V.T. Tikhonchuk ${ }^{4}$ \\ 1 LULI, Université Pierre et Marie Curie, 75005 Paris, France \\ 2 FNSPE, Czech Technical University, 11519 Prague, Czech Republic \\ ${ }^{3}$ CPHT, Ecole Polytechnique, 91128 Palaiseau, France \\ ${ }^{4}$ CELIA, Université Bordeaux 1, 33405 Talence, France
}

\begin{abstract}
Laser-plasma interaction is investigated for conditions relevant for the shock-ignition (SI) scheme of inertial confinement fusion using two-dimensional particle-in-cell (PIC) simulations of an intense laser beam propagating in a hot, large-scale, non-uniform plasma. The temporal evolution and interdependence of Raman- (SRS), and Brillouin- (SBS), side/backscattering as well as Two-Plasmon-Decay (TPD) are studied. TPD is developing in concomitance with SRS creating a broad spectrum of plasma waves near the quarter-critical density. They are rapidly saturated due to plasma cavitation within a few picoseconds. The hot electron spectrum created by SRS and TPD is relatively soft, limited to energies below one hundred $\mathrm{keV}$.
\end{abstract}

\section{SHOCK-IGNITION AND THE IMPORTANCE OF LASER-PLASMA INTERACTION}

The shock-ignition scheme [1] is based on an energy redistribution of the available energy: the laser pulse inducing compression of the pellet has lower intensity than in the original direct-drive scheme, followed by a second, intense laser pulse $(\sim 100 \mathrm{ps})$ which creates an intense short shock that combined with the rebound of the first shock allows the creation of a hot spot. The intensity of the second pulse is up to 10 times higher than the standard ones, and the laser will propagate in a hot (few $\mathrm{keV}$ ), long, inhomogeneous plasma. For laser plasma-interaction kinetic effects will be dominant.

A first estimate on the importance of laser plasma interaction comes from evaluating the local growth rate for parametric instabilities. As a consequence, even though the proposed intensities for the shock pulse are high, $I_{o} \lambda_{o}^{2}=10^{15 \ldots 16} \mathrm{~W} \mu \mathrm{m}^{2} / \mathrm{cm}^{2}$ at $3 \omega_{o}$, Stimulated Raman Scattering (SRS) was not expected to be a great danger since the pulse propagates in a hot plasma of $3 \ldots 5 \mathrm{keV}$ and the excited electron plasma waves by SRS are in a strongly damped regime. Some concern could come from the fact that a regime of inflationary SRS [2] was predicted, but the main worry was expected to originate from strong Stimulated Brillouin Scattering (SBS) developing along the whole density profile. 1D-simulation investigating this ansatz showed unexpected results [3]: after an initial phase SBS is suppressed by absolute SRS and cavitation at $n_{c} / 4$ and subsequent cascade at $n_{c} / 16$. As a result laser absorption is by collective effects and cavitation appears as a dominant mechanism. 2D-simulations confirm some of these results, but show a richer physics $[4,5]$. Plasma parameters and profiles are motivated by CHIC hydro-simulations of the HiPER target as considered in the 1D-simulations [3]. The simulation set up is as follows: the size of the plasma is $160 \mu \mathrm{m} \times 103 \mu \mathrm{m} ; T_{e}=5 \mathrm{keV}, T_{i}=1 \mathrm{keV}$; the density varies from $n=0.04 n_{c}$ to $n=0.4 n_{c}$, it has exponential profile with scale length $L_{n}=186 \lambda_{o} \approx 60 \mu \mathrm{m}$, followed

\footnotetext{
ae-mail: caterina.riconda@upmc.fr
}

This is an Open Access article distributed under the terms of the Creative Commons Attribution License 2.0, which permits unrestricted use, distribution, and reproduction in any medium, provided the original work is properly cited. 

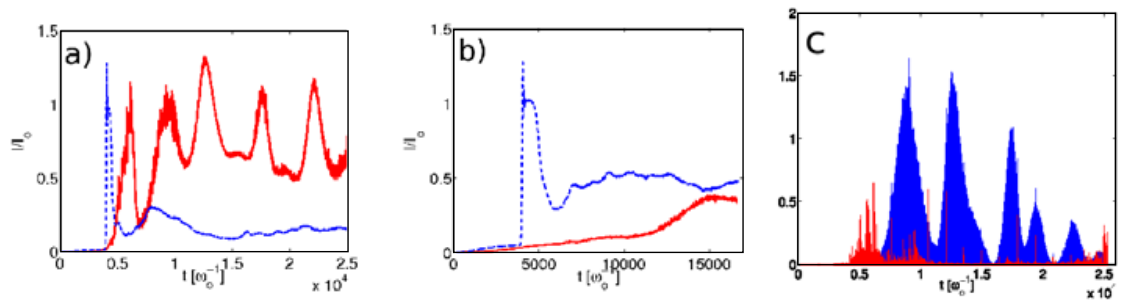

Figure 1. Reflectivity (in red) and transmission (in blue) for (a) $I_{o} \lambda_{o}^{2}=4.9 \times 10^{15} \mathrm{~W} \mu \mathrm{m}^{2} / \mathrm{cm}^{2}$, and (b) $I_{o} \lambda_{o}^{2}=$ $1.2 \times 10^{15} \mathrm{~W} \mu \mathrm{m}^{2} / \mathrm{cm}^{2}$. c) Frequence decomposition of the relectivity for the high intensity case: in blue contribution from SBS $\left(0.9-1.1 \omega_{o}\right)$ and in red from SRS $\left(0.0-0.9 \omega_{o}\right)$.

by a $\sim 15 \mu$ m plateau at $0.4 n_{c}$; the laser has a gaussian shape in the transverse direction with FWHM $\approx 29 \mu \mathrm{m}$ and intensity at the maximum given by $I_{o} \lambda_{o}^{2}=1.2-4.9 \times 10^{15} \mathrm{~W} \mu \mathrm{m}^{2} / \mathrm{cm}^{2}$ at $3 \omega_{o}$. The total simulation time is $\approx 2.5 \times 10^{4} \omega_{o}^{-1} \approx 5 \mathrm{ps}$.

\section{REFLECTIVITY DATA FOR THE FULL SPECKLE}

Figures 1a,b show the $k_{\perp}$-integrated reflectivity (in red) and transmission (in blue). It is clearly visible that the transmission is low and "steady-state" in both cases for the time scales considered. As the reflectivity levels are not high enough to justify the low transmission, one can deduce that significant non-collisional absorption of the incoming laser takes place in the first picoseconds. As will be shown later most of the absorption is at or below the quarter-critical density, contrary to the current idea that absorption is predominantly by inverse Bremsstrahlung at the critical density. Another remarkable feature, evident especially in the high intensity case, is the presence of reflectivity bursts due to SBS by independent filament activity. Analysis of the poynting vector shows that the original laser speckle quickly breaks into filaments, while analysis of the ion density shows that Brillouin develops at $n_{e}<0.2 n_{c}$, and successive burst of Brillouin reflectivity come from different filaments. In the highest intensity case, $I_{o} \lambda_{o}^{2}=4.9 \times 10^{15} \mathrm{~W} \mu \mathrm{m}^{2} / \mathrm{cm}^{2}$ inflationary Raman at $n_{e}<n_{c} / 4$ is observed, but does not contribute significantly to the overall energy balance. In general one can observe that reflectivity levels decrease considerably with decreasing $I_{o}[4,5]$. Figure 1c displays the value of $B_{r e f}^{2} / B_{o}^{2}$ at the center of the speckle, frequency decomposed, as a function of time for the high intensity case. Here $B_{r e f}$ indicated the magnetic field of the reflected light, while / $B_{o}$ indicated the incoming light. This diagnostic allows to infer the contribution of SBS and SRS to the integrated reflectivity. It shows that the evolution of SRS and SBS depends on the considered location. Although the average reflectivity still has large SBS burst (see Fig.1a), one can deduce that the late time bursts come from the wings of the speckle, while at the center SBS is saturating at lower values. Analysis of the SBS and SRS spectrum at the center and in the wings of the speckle is consistent with the idea that the instability evolves independently in space and coherently with the local growth rates. For example the spectrum in the wings of the speckle (lower intensity) is less broad than in the center, and grows more slowly with time, so that it is still increasing while in the center there is already saturation. This is also confirmed by analysis of density fluctuations related to SBS as a function of space and time. As far as SRS is concerned instead, it is observed that SRS activity is strong initially, comes mainly from close to $n_{c} / 4$ and reappears later on in the low density ramp, with occasional bursts at $n_{c} / 4$. Backscattering inside a speckle is strongly space- and timedependent resulting for SRS in a very large spectrum for the backscattered wave, that has the signature of where SRS developed. Figure 2 reproduces the time evolution of the SRS spectrum as a function of the transverse location, for four time intervals for the high intensity case. The intervals are chosen early in the simulation: $3106-\mathbf{4 6 4 2} \omega_{0}^{-1}$ (a) and $6108-\mathbf{7 6 0 9} \omega_{0}^{-1}$ (b), and towards the end of the simulation: $16405-17941 \omega_{\mathbf{0}}^{-1}$ (c) and $23806-25272 \omega_{\mathbf{0}}^{-1}$ (d). A filamented structure of the original 

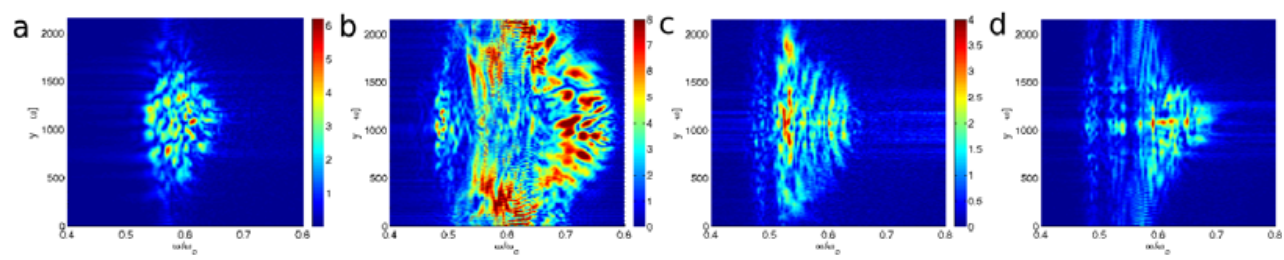

Figure 2. a-d) Spectrum of the backscattered light in the interval $0.4-0.8 \omega_{0}$ as a function of the transverse direction.
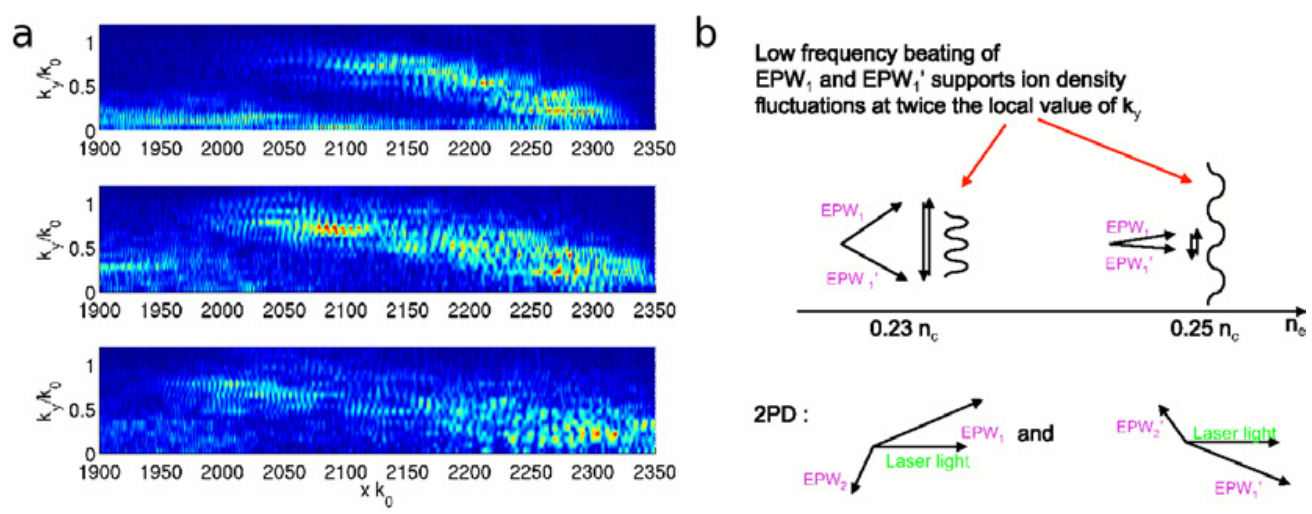

Figure 3. a) Snapshots of $k_{\perp} / k_{o}$ of the electron plasma wave excited around $n_{c} / 4$ as a function of space. Quarter critical density is located at $\sim x k_{o}=2300$. Times are (top to bottom) $t \omega_{o}=3000,3400,4000$. b) Geometry of the plasma wave beating and TPD.

speckle is observed which shows SRS-activity in distinct regions. The actual region of activity in the plasma profile follows directly from the frequency of the backscattered light. In particular, if $n \ll n_{c}$ one can directly deduce the location form the formula $\omega_{1} / \omega_{o} \approx 1-\sqrt{n / n_{c}}$. Activity in the frequency range $0.7 \ldots 0.8 \omega_{o}$ is a strong indication of inflationary Raman. In agreement with the integrated reflectivity results the late time SRS activity is strongly reduced, and after occasional bursts around $0.25 n_{c}$, SRS retreats to the low density region.

\section{TWO-PLASMON-DECAY INSTABILITY}

TPD is driven far above absolute threshold, $I_{14} \lambda_{o}^{2}>51 \frac{T e[k e V]}{\left(L / \lambda_{o}\right)}$, so that it could be responsible of producing hot electrons. Distinctive signatures of TPD have been observed in the simulations such as $3 / 2 \omega_{o}$-signal in the transmitted light and distinctive values of $k_{\perp}$ of the electron plasma waves corresponding to optimal growth rate at different space location (see Fig. 3a)[6]. The time evolution of the electron plasma wave spectrum, Fig. 3a, and of the transmitted light signal provide evidence for fast saturation of the TPD instability. As a result the electrons produced in the process are not "too" hot, and their production is limited in time. The TPD saturation is related to the appearance of quasineutral density fluctuations driven by the low frequency beating of electron plasma waves generated by the TPD process evolving into cavities[4, 7, 8] (see schematic representation in Fig. 3b). Close to $n_{c} / 4$ the electron plasma waves have small perpendicular wavevectors resulting in long wavelength beating, while further away from $n_{c} / 4$ the perpendicular wavevectors are larger resulting in smaller wavelength. This is confirmed by Fourier analysis of the ion density fluctuations (not shown here), and by a zoom of the ion density fluctuations around $n_{c} / 4$ : purely transverse regular fluctuations are clearly observable in Fig. 4a with increasing wavelength as one gets closer to $n_{c} / 4$ (located at $x k_{o} \sim 2300$ ). 
a

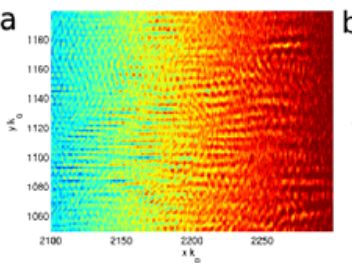

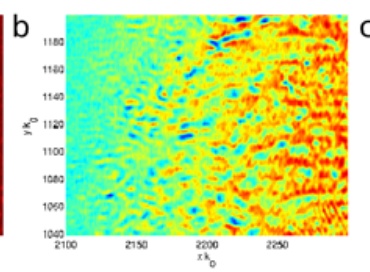

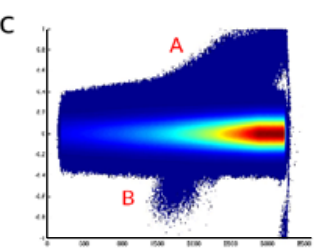

Figure 4. a) Zoom of the ion density from the high I case at $t \omega_{o}=3400$ showing the structures originating from the plasma wave beating. b) Zoom of the ion density from the high I case at $t \omega_{o}=6500$ with cavities well established. Parallel (c) and perpendicular (d) electron phase space at $t \omega_{o}=4800$.

Their interaction with the downshifted backscattered SRS-light make the transverse fluctuations evolve into cavities of trapped intense electromagnetic structures, as shown in Fig. 4b. Backscattered SRS light from close to $n_{c} / 4$ has the right frequency to be locally trapped by the ambient density in the presence of fluctuations[4]. A possible hybrid solution in competition with simple TPD or SRS, the 'HighFrequency-Hybrid-Instability', might contribute to the modes observed [9]. Once the first fluctuations are generated, cavity creation and disappearance is a continouus process in the vicinity of $n_{c} / 4$, and they are responsible for continuous heating without a preferential direction. Moreover, as a result of cavity creation, a dynamic RPP (random phase plate) is created around the quarter-critical density extending over a distance of few $100 k_{o}^{-1}$. Behind this layer much smaller filamentary structures with large opening angle appear $[4,5]$. Figure $4 \mathrm{c}$ and $4 \mathrm{~d}$ present the electron phase space for velocities parallel and perpendicular to the laser propagation direction at time $t \omega_{o}=4800$ when hot electrons produced by Raman, TPD, and cavities are present: 'A' labels electrons going into the forward direction (laser propagation direction). The main contribution to this bunch can be related to electrons associated with plasma waves generated by the Raman instability, that have a very small opening angle. Electrons associated to TPD created plasma waves and to stochastic heating by the localized fields in the cavities are also present. However, the latter, having much less energy, are not apparent as a separate population in the figure. The label ' $\mathrm{B}$ ' refers to electrons going in the backward direction: they are mainly associated to TPD-produced plasma waves (see scheme in Fig. 3b) and cavities. In particular the less energetic ones in this group, that are closer to $n_{c} / 4$, are interpreted as being related mainly to cavities. By symmetry, there is no preferential direction perpendicular to the laser propagation direction. Electrons produced by Backward Raman Scattering will have very small, or zero, value for the perpendicular velocity component, and thus are not distinguishable from the bulk. The energetic electrons labeled as ' $\mathrm{C}$ ' are thus interpreted as being produced by the TPD electron plasma waves moving towards the low density part (associated to particles in 'B'), and the electrons labeled as ' $\mathrm{D}$ ' as the ones associated to TPD plasma waves moving forward and cavities. The averaged electron distribution function at early time is consistent with this picture, while at later time it shows indeed slow continuous heating of the electrons with few very hot (more than $50 \mathrm{keV}$ ) electrons $[4,5]$.

\section{CONCLUSIONS}

The interplay of many mechanism, as presented here, shows the need of multi-dimensional, large, fully kinetic simulations for long times. Fast saturation of TPD and SRS close to $n_{c} / 4$ is observed by cavity formation. The cavities of trapped light are seeded by beating of TPD-generated electron plasma waves. As a result electron heating in the low density plasma produces very hot electrons only for a limited amount of time, and the resulting electron distribution function has a typical temperature of $30-50 \mathrm{keV}$. The creation of a dynamic RPP is observed at $n_{c} / 4$ (see Fig. 4b) that will affect the long time evolution of the parametric instabilities. 


\section{IFSA 2011}

\section{References}

[1] R. Betti et al., Phys. Rev. Lett., 98, 155001 (2007)

[2] H. Vu et al., Phys. Rev. Lett., 86, 4306 (2001)

[3] O. Klimo et al., Plasma Phys. Control. Fusion, 52, 055013 (2010)

[4] C. Riconda et al., Phys. Plasmas, 18, 092701 (2011)

[5] S. Weber et al., Phys. Rev. E, 85, 016403 (2012)

[6] R. Yan et al., Phys. Rev. Lett., 103, 175002 (2009)

[7] A. Langdon et al., Phys. Rev. Lett., 43, 133 (1979); A. Langdon et al. Phys. Fluids, 26, 582 (1982)

[8] S. Weber, et al. Phys. Rev. Lett., 94, 055005 (2005); C. Riconda et al., Phys. Plasmas, 13, 083103 (2006)

[9] B. B. Afeyan, E. A. Williams, Phys. Rev. Lett.,75, 4218 (1995); Phys. Plasmas, 4, 3845 (1997) 